\title{
INTERDISCIPLINARY APPROACH FOR FULL MOUTH REHABILITATION: A CASE REPORT
}

\author{
ZAID AL-JEAIDI*
}

Department of Conservative Dental Science, College of Dentistry, Prince Sattam Bin Abdul Aziz University, Al Kharj 11942, Kingdom of Saudi Arabia. Email: dr.aljeaidi@gmail.com

Received: 26 July 2016, Revised and Accepted: 08 August 2016

\section{ABSTRACT}

The demand for esthetic dentistry is ever increasing. A beautiful smile is considered as a symbol of health and vitality is now looked on as a status symbol. Discoloration of the front tooth may severely affect the confidence of the patient. The use of dental implant presents a conservative and esthetic method of replacing missing teeth. Bleaching is recommended as a minimal invasive and cost-effective approach to change the color of the teeth. This case reports the step by step procedure for full mouth rehabilitation of an esthetically demanding patient.

Keywords: Bleaching, Composites, Esthetics, Implant, Root canal treatment.

(C) 2016 The Authors. Published by Innovare Academic Sciences Pvt Ltd. This is an open access article under the CC BY license (http://creativecommons org/licenses/by/4. 0/) DOI: http://dx.doi.org/10.22159/ajpcr.2016.v9i6.14324

\section{INTRODUCTION}

The outcome of a case involving esthetic rehabilitation of the patient is dependent on the interdisciplinary approach and understanding [1]. Every patient visiting the dental office brings with him higher level of esthetic expectations and requires thorough knowledge and adequate clinical expertise to fulfill such a requirement efficiently $[2,3]$. This unprecedented improvement in esthetic rehabilitation has occurred due to advancements in restorative materials and techniques [4].

Traumatic injuries are the most common in upper anterior teeth which compromise both esthetics and function [5]. Such injuries can result in fracture of teeth at different levels with varying difficulties of restorability and can also result in loss of vitality of the tooth and discoloration [6,7]. The cause for discoloration may be intra pulpal hemorrhage and pulp necrosis [8].

Bleaching of discolored teeth either vital or nonvital is a popular esthetic approach which is conservative and gives good results [9]. Over the years, various bleaching agents have been used with varying results. For vital teeth, In-office tooth bleaching is a popular technique which uses hydrogen peroxide in high concentrations [10-12]. For non-vital endodontically treated teeth, inside/outside bleaching technique is an effective option [13-15].

In this case, a combined approach was used simultaneously wherein the vital teeth were bleached using Opalescence Boost PF 40\% (Ultra Dent Product, Inc. USA) containing $40 \%$ hydrogen peroxide which is activated chemically and the discolored non-vital teeth was root canal treated and bleached using the same product by inside/outside bleaching technique.

The rehabilitation of partial or total edentulous area by dental implant is a conservative and predictable means of replacing missing teeth $[16,17]$. In the present case, a two-stage implant procedure was performed. During the first stage, the implant was inserted and in the second stage after 6 months prosthetic abutment was attached.

\section{CASE REPORT}

A 28-year-old male Sudanese patient reported to the dental clinic at College of Dentistry, Prince Sattam Bin Abdulaziz University, Alkharj, with a chief complaint of unaesthetic appearance of front teeth and replacement of missing teeth (Fig. 1). The medical history of the patient was not significant. He had a past dental history of extractions in the upper left and right back region due to caries. Thorough clinical examination revealed occlusal caries in relation to teeth 17,16 and 37 ; proximal caries in relation to teeth 16,15 and 26; temporary restoration in relation to tooth 47 , missing teeth 14 and 25 , Ellis Class III fractured 21 and Class II fracture with 12 .

An orthopantomograph was advised along with intra oral periapical (IOPA) radiograph with teeth 21, 12 and 47 and bitewing radiograph with teeth 16, 15 and 26 (Fig. 2).

Ethyl chloride spray (Gebauer, Cleveland, Ohio, USA) was used for pulp testing in relation to 12 and 21.12 revealed signs of irreversible pulpitis and was diagnosed as asymptomatic apical periodontitis and 21 showed no response. IOPA revealed a large periapical lesion and was diagnosed as pulp necrosis. IOPA with tooth number 47 revealed previous attempted incomplete root canal treatment.

Hence, a treatment plan was made keeping the patients chief complaint in mind. It was aimed to eliminate pathosis, improve esthetics, and restore the function.

\section{Treatment plan}

- Oral prophylaxis

- Caries removal and glass ionomer interim restorations.

- Root canal treatment.

- Implant supported prosthesis.

- Vital and nonvital tooth bleaching.

- Composite restorations

- Full coverage restorations.

\section{Treatment sequencing}

Phase 1

Oral prophylaxis was performed in a single visit. For tooth numbers - 17, 16, 15, 26 and 37, caries control and interim glass ionomer cement restorations (Fugi IX, GC America) were done.

Phase 2

Root canal treatment was performed with teeth 12, 21 and 47 and restored with glass ionomer cement. 


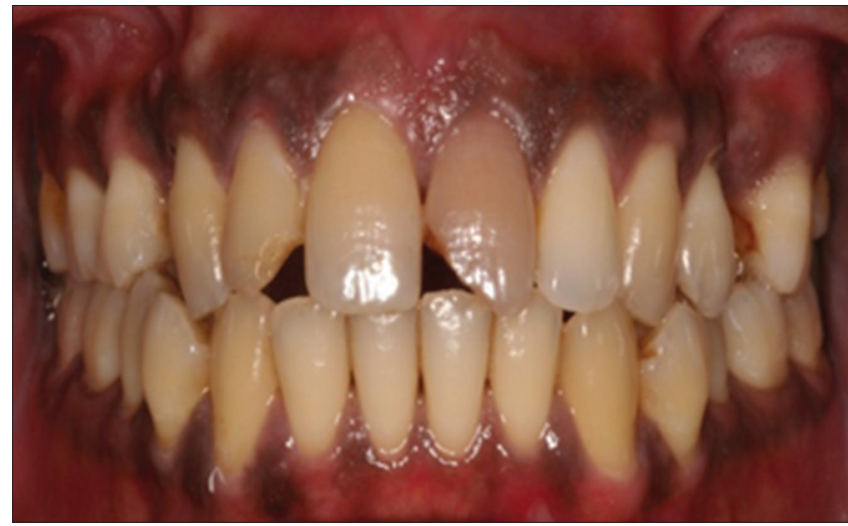

Fig. 1: Pre-operative intraoral photograph

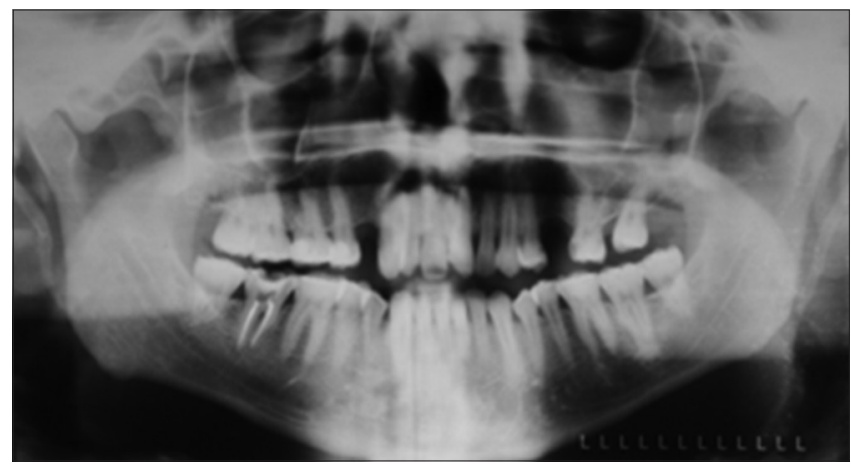

Fig. 2: Pre-operative orthopantomograph

Phase 3

Two Nobel Replace Implants (Nobel Biocare, Zurich, Switzerland) were placed surgically in the edentulous area according to the guidelines for implant placement. After implant insertion, the corresponding healing screws were threaded into position (Figs. 3 and 4).

\section{Phase 4}

The teeth were dried and Opal Dam (Ultradent Product, Inc. USA) was applied on the gingival margin and overlapping approximately $0.5 \mathrm{~mm}$ on the enamel from tooth numbers $16-26$ and light cured for 20 seconds. This was followed by the application of 40\% Opalescence Boost PF (Ultradent Product, Inc. USA), bleaching agent to the labial surfaces for 20 minutes. The gel was suctioned using high vacuum without water, and the procedure was repeated 3 times. For 21, the gutta-percha was removed $2 \mathrm{~mm}$ below the orifice level and a glass ionomer barrier was placed. Using $37 \%$ phosphoric acid, pulp chamber was etched for 30-60 seconds, washed and dried, which resulted in the opening of dentinal tubules. Following this, 40\% Opalescence Boost PF (Ultradent Product, Inc. USA) bleaching agent was mixed into thick paste and placed immediately in the pulp chamber and on the external labial surface of the tooth. After 10-15 minutes, the tooth was cleansed and the residue bleach inside was removed with water using a high vacuum suction unit. The procedure was repeated three times. Following the final wash, tooth shade was evaluated, which matched with adjacent tooth and satisfactory results were achieved. There was a significant improvement in the appearance of the teeth and a shade of B2 was achieved. The access and the partially empty pulp chamber were restored using B2 composite resin. B2 shade microhybrid composite (3M ESPE) was used to fill the teeth permanently which were previously restored with glass ionomer cement.

\section{Phase 5}

After a healing period of 6-month as advised in the classical Brannemark 2 stage submerged protocol, a tissue punch was used to uncover the

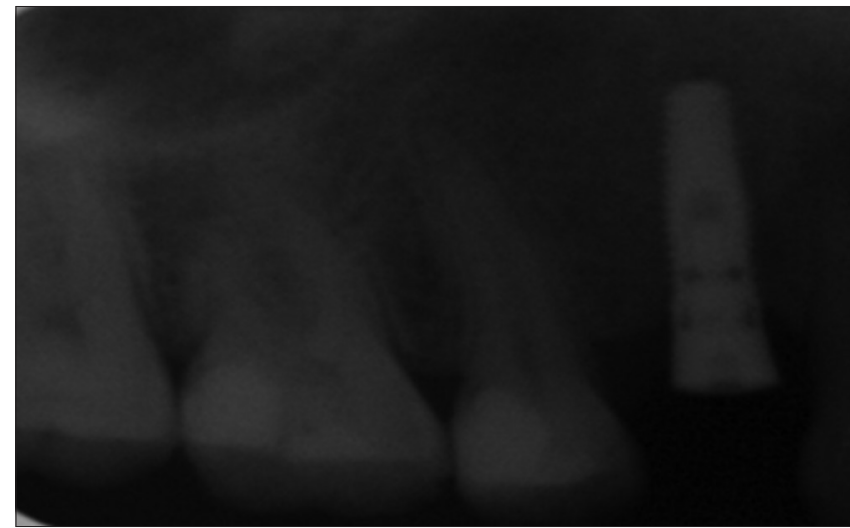

Fig. 3: Implant placed in edentulous space area related to tooth 14

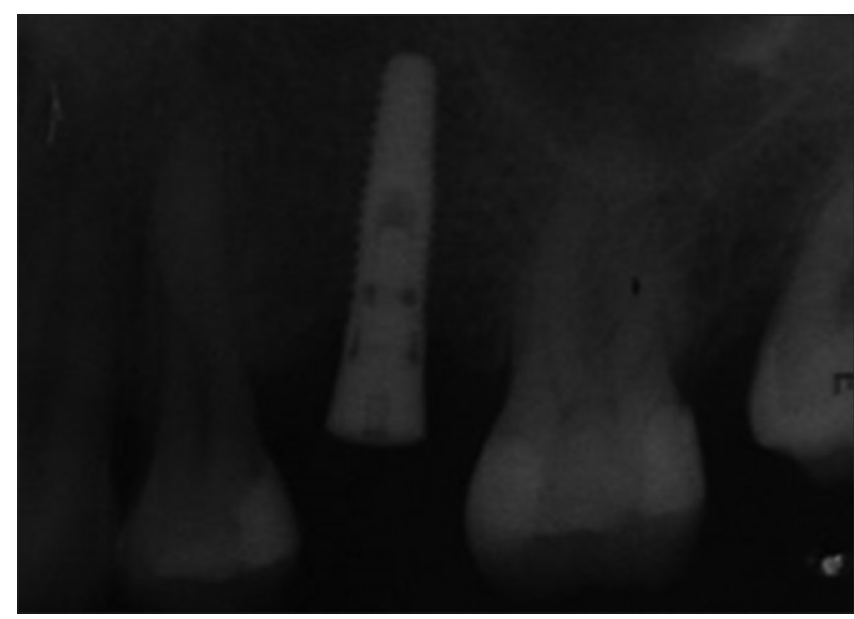

Fig. 4: Implant placed in edentulous area related to tooth 25

implant. The covering screws were removed and replaced by gingival former. After 2 weeks, the gingival former was removed and transfer coping and a transfer cap were placed. Impression was made with light body around the transfer coping and heavy body for the rest of the dentition (Figs. 5 and 6). Final full coverage restoration was done using an IPSe. Max Press (Ivoclar Vivadent) (Figs. 7 and 8).

\section{DISCUSSION}

A meticulous treatment plan is necessary when dealing with the case of full mouth oral rehabilitation. In this case, the treatment plan was formulated carefully depending on the patients need.

Opalescence tooth whitening gel contains PF (potassium nitrate and fluoride). Potassium nitrate has been shown to reduce sensitivity. Fluoride helps to reduce caries and strengthen enamel. It is available as a gel containing $40 \%$ hydrogen peroxide. Its use has been well documented in vital bleaching, but in this case, it is also used for nonvital bleaching taking necessary precautions to prevent cervical erosion as seen in other case reports [18].

Advances in technology have altered our treatment philosophy in the replacement of a single tooth. In many instances, a single-tooth implant is the restoration of choice, providing a highly esthetic, functional, and long-term result. The tremendous advantage of the single-tooth implant lies in the fact that the adjacent teeth are not prepared. These teeth are left in their current state of health and are not linked as part of a larger restoration. The adjacent teeth have a better prognosis, as they are not subject to a higher incidence of endodontic therapy and decay as a result of tooth preparation [19]. 


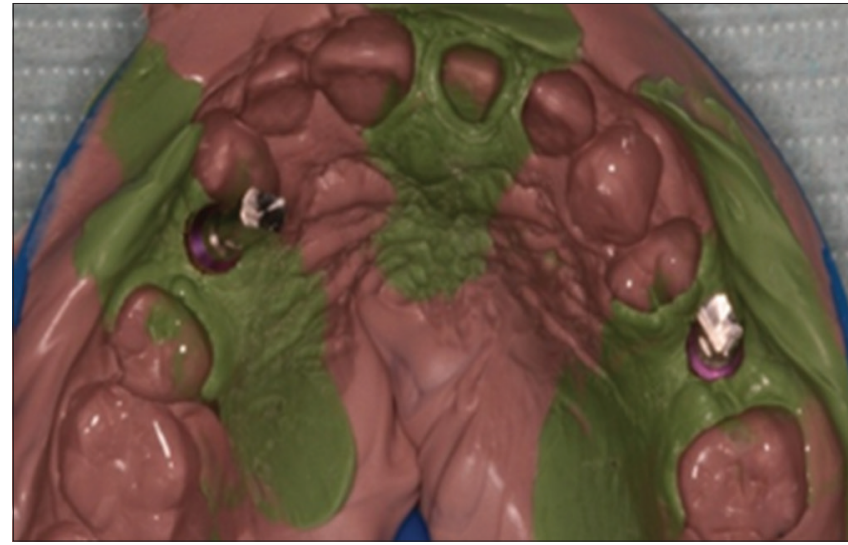

Fig. 5: Impression

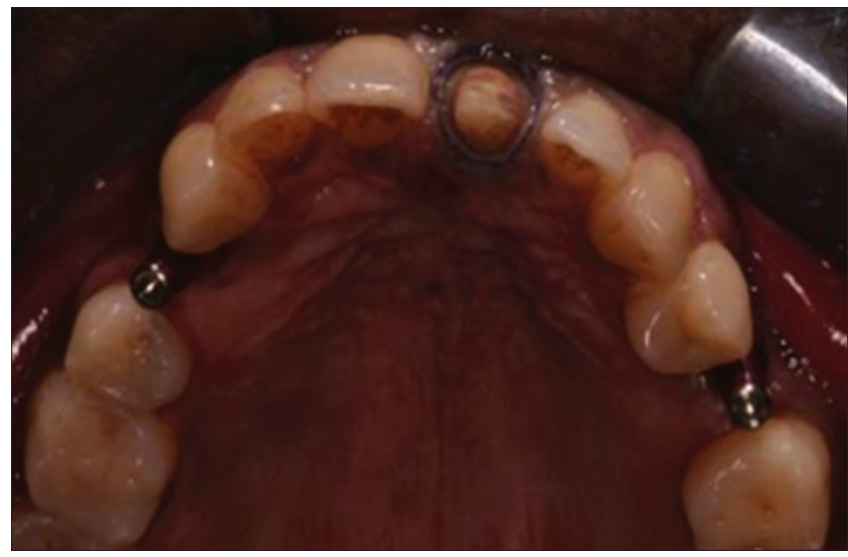

Fig. 6: Implant abutments placed

Hence, full mouth rehabilitation with interdisciplinary approach is necessary to eliminate pathosis and restore the function for esthetically demanding patient.

\section{REFERENCES}

1. Spear FM, Kokich VG, Mathews DP. Interdisciplinary management of anterior dental esthetics. J Am Dent Assoc 2006;137(2):160-9.

2. Flanagan D. Complete artificial dentition supported by endosseous implants: A case report of total in-office treatment. J Oral Implantol 2005;31(2):91-7.

3. Naveen BH, Kashinath KR, Mythri H, Nausheen H. Rare but reality of methyl methacrylate in dentistry - A case study. Int J Pharm Pharm Sci 2014;6(6):39-40.

4. Patzer GL. Understanding the causal relationship between physical attractiveness and selfesteem. J Esthet Dent 1996;3:144-6.

5. Alani A, Austin R, Djemal S. Contemporary management of tooth replacement in the traumatized dentition. Dent Traumatol 2012;28(3):183-92.

6. Hattab FN, Qudeimat MA, Al-Rimawi HS. Dental discoloration: An overview. J Esthet Dent 1999;11(6):291-310.

7. Olsburgh S, Jacoby T, Krejci I. Crown fractures in the permanent dentition: Pulpal and restorative considerations. Dent Traumatol 2002;18(3):103-15

8. Nathoo SA. The chemistry and mechanisms of extrinsic and intrinsic

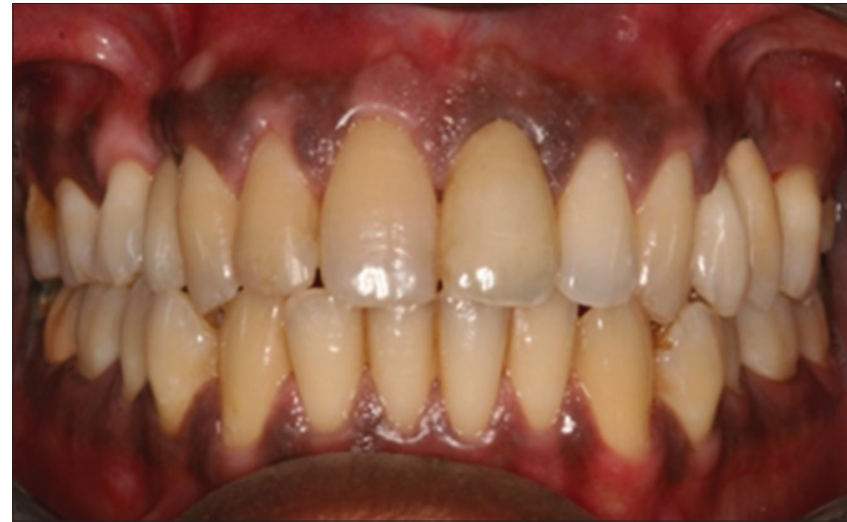

Fig. 7: Final post-operative intra oral photograph

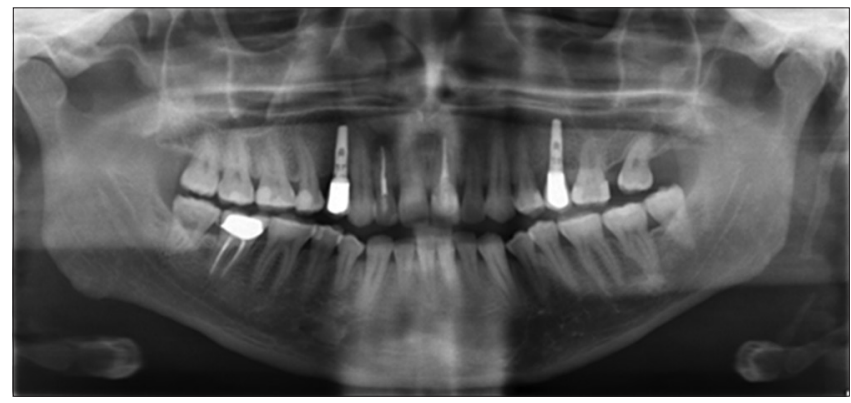

Fig. 8: Final post-operative orthopantomograph

discoloration. J Am Dent Assoc 1997;128 Suppl:6S-10.

9. MacIsaac AM, Hoen CM. Intracoronal bleaching: Concerns and considerations. J Can Dent Assoc 1994;60(1):57-64.

10. Luk K, Tam L, Hubert M. Effect of light energy on peroxide tooth bleaching. J Am Dent Assoc 2004;135(2):194-201

11. de Silva Gottardi M, Brackett MG, Haywood VB. Number of inoffice light-activated bleaching treatments needed to achieve patient satisfaction. Quintessence Int 2006;37(2):115-20.

12. Tavares M, Stultz J, Newman M, Smith V, Kent R, Carpino E, et al. Light augments tooth whitening with peroxide. J Am Dent Assoc 2003;134(2):167-75.

13. Leith R, Moore A, O'Connell AC. An effective bleaching technique for non-vital, discoloured teeth in children and adolescents. J Ir Dent Assoc 2009;55(4):184-9.

14. Attin T, Paqué F, Ajam F, Lennon AM. Review of the current status of tooth whitening with the walking bleach technique. Int Endod J 2003;36(5):313-29.

15. Settembrini L, Gultz J, Kaim J, Scherer W. A technique for bleaching nonvital teeth: Inside/outside bleaching. J Am Dent Assoc 1997;128(9):1283-4.

16. Klokkevold PR, Newman MG. Current status of dental implants: A periodontal perspective. Int $\mathrm{J}$ Oral Maxillofac Implants 2000;15(1):56-65.

17. SchellerH,UrgellJP,KultjeC,KlinebergI,GoldbergPV,Stevenson-MooreP, et al. A 5-year multicenter study on implant-supported single crown restorations. Int J Oral Maxillofac Implants 1998;13(2):212-8.

18. Walton RE, Rotstein L. Bleaching discolored teeth: Internal and external. Principles of Endodontics. $2^{\text {nd }}$ ed. Ch. 23. Philadelphia, PA: W. B. Saunders; 1996. p. $385-400$

19. Hebel K, Gajjar R, Hofstede T. Single-tooth replacement: Bridge vs. Implant-supported restoration. J Can Dent Assoc 2000;66(8):435-8. 